\title{
VARIATIONS IN DENTAL ARCH MORPHOLOGY ARE OUTCOMES OF THE COMPLEX ADAPTIVE SYSTEM ASSOCIATED WITH THE DEVELOPMENTAL VARIATION OF HYPODONTIA
}

\author{
DILAN PATEL ${ }^{1}$, SADAF SASSANI $^{1}$, MAURO FARELLA ${ }^{2}$, SARBIN RANJITKAR $^{1}$, ROBIN YONG $^{1}$, \\ STEVE SWINDELLS ${ }^{2} \&$ ALAN BROOK $^{1,3}$ \\ ${ }^{1}$ Adelaide Dental School, the University of Adelaide, Australia. \\ ${ }^{2}$ Faculty of Dentistry, University of Otago, New Zealand. \\ ${ }^{3}$ Institute of Dentistry, Queen Mary University of London, United Kingdom.
}

\begin{abstract}
Development of the human facial structures including the jaws and dentition occurs in a process that has the characteristics of a complex adaptive system (CAS) influenced by epigenetic, genetic and environmental factors. Earlier studies have suggested dental arch development to be reduced in size in subjects with hypodontia when compared with controls. Hypodontia is a variation of development and presents with a reduced number of teeth together with several other phenotypic changes. This study uses enhanced 3D imaging techniques to increase the accuracy of the measurements of dental arches. The sample consists of orthodontic patients, 60 with hypodontia (thirty males and thirty females), and 60 controls matched for age, gender and ethnicity. One operator using an Amann Girrbach Ceramill Map400 3D scanner recorded the 3D images from dental models. The 3D images were then viewed on MeshLab and the accuracy of the measurements were determined through repeat measurement of the same images; this was undertaken with intra- and inter-operator reproducibility. Ten repeat measurements were taken on 10 different models. Validation of the new system was undertaken by repeating the measurements using the standard 2D caliper technique. Arch dimension measurements were determined from distance between the left-hand side first molar to the right-hand side first molar. Similar measurements were also made for the inter-canine width. The results for average intraoperator measurements were $0.33 \mathrm{~mm}$ for the maxillary arch and $0.40 \mathrm{~mm}$ for the mandibular arch. The difference in average inter-operator reproducibility was also measured for inter-molar arch dimensions at 0.31 and $0.23 \mathrm{~mm}$ for maxillary and mandibular arches, respectively. This novel method provides an increased range of measurement of similar accuracy to standard techniques. This study will proceed to establish the variations on the 3D images between the hypodontia subjects and the control group.
\end{abstract}

Keywords: 3D imaging, dental arch, hypodontia, measurements, morphology, reliability, repeatability.

\section{INTRODUCTION}

Hypodontia is largely considered to be a variation of dental development with up to 6 congenitally missing teeth excluding third molars. It forms part of the complex adaptive system (CAS) of oro-facial development whereby epithelial tissues and neural crest mesenchyme are involved in delicate signalling pathways with multiple molecular events that overlap each other [1-4]. For a system to be considered as complex and adaptive, it should exhibit the principles of self-organisation and self-adaptation. The features of self-organisation and emergence occur during dental development at the initiation and morphogenetic stages. Separate genetic pathways and genetic/epigenetic/environmental interactions occur simultaneously throughout the dental developmental process; this is evidence of multitasking. The intricate and multilevel processes that occur during odontogenesis demonstrate the characteristics of a CAS $[1,5-8]$. 
Hypodontia is widely reported to be the most common craniofacial malformation in humans with varying reports of between $2 \%$ and $10 \%$ of the global population in the permanent dentition excluding third molars [8-11].

The dental arch consists of the dentition and the surrounding structures; alveolar bone forms a large component of the tissues surrounding the dentition. Development of the alveolus is influenced by the presence of teeth. This forms a part of the CAS; however, there is a marked environmental influence during development and function $[5,7,8]$.

\section{AIM}

Dental plaster study models of the maxillary and mandibular dentition have for several decades been used by dental practitioners as a vital aid in treatment planning and form part of the patients' clinical and medico-legal records. Plaster models are especially relevant for orthodontic patients, and many patients with hypodontia require orthodontic treatment as part of the multidisciplinary treatment planning. Plaster models are disadvantageous in that they are relatively delicate and require careful storage and labelling. When many models are collected over a long period, they can take up a considerable amount of space which also incurs a cost. Additionally, when measuring and using models over a long period they are subjected to a degree of wear and tear, which can alter the measurements [12-16].

Three-dimensional scanning has been developed as a recent method for measuring and assessing dental models. With the development of scanning technologies, it has become increasingly relevant to apply this technology to the dental arch and test the accuracy of measuring dental scans between operators [14].

The aim of this paper is to determine the accuracy of 3D scanning technologies in assessing arch dimensions as part of a multicentre, multidisciplinary collaborative study of hypodontia relating genotype and phenotype.

\section{MATERIALS AND METHODOLOGY}

This study has a unique sample group with defined parameters. The 120 subjects are orthodontic patients from the University of Otago. The hypodontia group was matched for age, gender and ethnicity with equivalent controls. Thirty male hypodontia subjects are in the age range of 10.2-21.0 years old and matched with 30 controls in the age range of 10.1-17.3 years old (Table 1). Thirty female hypodontia subjects are in the age range of 10.8-19.3 matched with controls in the age range of 10.6-19.2 (Table 1). This is summarised in Table 1. The ethnicity of the sample was self-identified by each of the subjects who were matched together. A statistically relevant number of hypodontia and control subjects were recorded [15].

Duplicate die stone models were poured and trimmed from existing models of the maxillary and mandibular dental arches of each subject in the study. Each model was placed in a fixed repeatable position in an Amann Girrbach Ceramill Map400 3D scanner, with the scanner set on its highest detection limit of 20 microns.

Table 1: Study sample of hypodontia patients matched with controls for gender and age.

\begin{tabular}{lll}
\hline & Age range of subjects (in years) & \\
\hline Number in each gender & 60 Males & 60 Females \\
Hypodontia & $10.2-21.0$ & $10.8-19.3$ \\
Controls & $10.1-17.3$ & $10.6-19.2$ \\
\hline
\end{tabular}




\subsection{Validation}

Caliper measurements of the same point-to-point measurements were recorded on the dental models using a Craftright 150-mm stainless steel digital Vernier caliper to validate the 3D scans.

\subsection{Measurements}

To assess the molar width of the arches, measurements of the 3D scans were recorded on the maxillary arch from the disto-buccal cusp tip of tooth 16 to the corresponding cusp on tooth 26 (Fig. 1). Similarly, on the mandibular arch, the distance was measured between the distobuccal cusp of tooth 36 and the disto-buccal cusp of tooth 46 (Fig. 2). Ten measurements of each arch were recorded from the control group of this preliminary study.

The MeshLab software program was used to record measurements; it is a relatively simple mesh processing system and is often used in processing meshes from 3D scans. The software was chosen for its ease of use in recording simple point-to-point measurements including the ability to rotate the model in any direction, while taking a measurement, this assists in ensuring the highest point of the cusp tip is recorded.

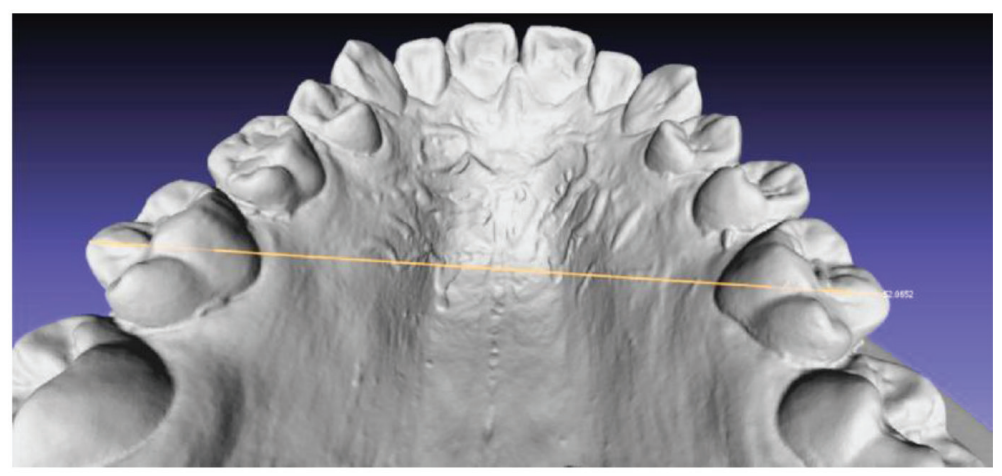

Figure 1: Maxillary inter-molar measurement, magnified view.

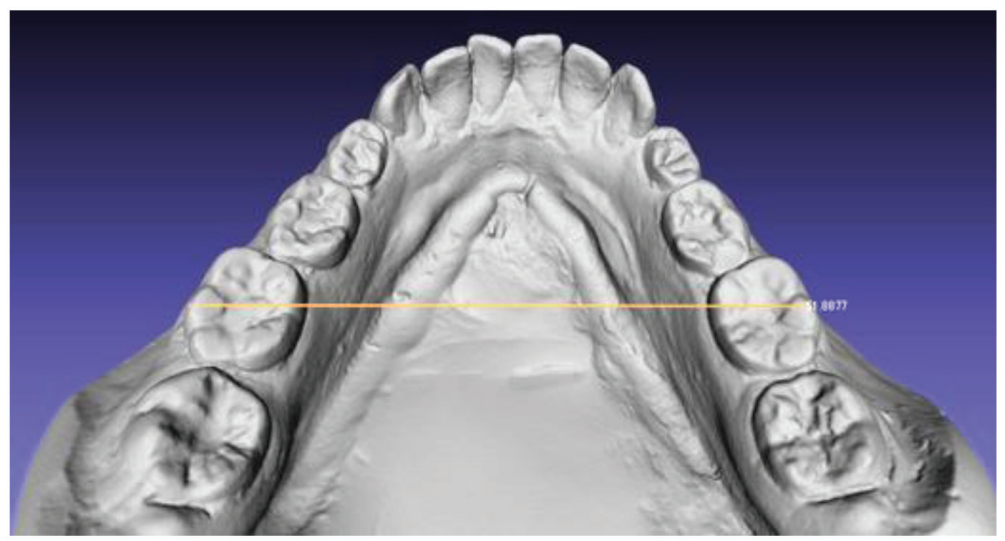

Figure 2: Mandibular inter-molar measurement, full arch view. 


\subsection{Error determination and repeatability testing}

Variability in the measurement process results in multiple repeat measurements of the same distance. In instances where the molar cusp tip was not clearly defined or there was significant wear of the cusp, the reference points for the linear measurement may cause greater variance. Technical error of measurement (TEM) should be considered to determine if a statistically significant difference between repeat measurements exists. To quantify the TEM, a two-way random effect statistical model with single measure and absolute agreement was applied to the data. An intraclass correlation coefficient (ICC) model was used to calculate the above measurements using SPSS software for statistical analysis. To test for intra-operator error, these measurements were repeated 8 weeks later by the same operator (Operator 1). Additionally, a second operator with equal experience in using the MeshLab software (Operator 2) performed the same measurements to test for inter-operator error $[15,16]$.

\section{RESULTS}

The results for intra and inter-operator error are given in Tables $2-5$ below. All measurements listed are graded in millimetres ( $\mathrm{mm}$ ).

Table 2: Intra-operator error in the maxillary arch (in $\mathrm{mm}$ ).

\begin{tabular}{lll}
\hline Measurement time 1 & Measurement time 2 & Differential \\
\hline 51.38 & 51.54 & 0.16 \\
53.06 & 53.63 & 0.57 \\
58.19 & 58.54 & 0.35 \\
49.14 & 49.75 & 0.61 \\
57.72 & 57.89 & 0.17 \\
48.84 & 49.59 & 0.75 \\
56.21 & 56.01 & 0.20 \\
54.34 & 54.53 & 0.19 \\
51.88 & 51.66 & 0.22 \\
45.02 & 44.98 & 0.04 \\
& & Mean: 0.33 \\
\hline
\end{tabular}

Table 3: Intra-operator error in the mandibular arch (in $\mathrm{mm}$ ).

\begin{tabular}{lll}
\hline Measurement time 1 & Measurement time 2 & Differential \\
\hline 54.82 & 54.91 & 0.09 \\
46.69 & 46.61 & 0.08 \\
53.06 & 52.52 & 0.54 \\
43.17 & 44.35 & 1.18 \\
51.18 & 51.39 & 0.21 \\
42.89 & 43.62 & 0.73 \\
49.26 & 48.88 & 0.38 \\
47.99 & 48.45 & 0.46 \\
45.13 & 45.49 & 0.36 \\
40.21 & 40.20 & 0.01 \\
& & Mean: 0.40 \\
\hline
\end{tabular}


Table 4: Inter-operator error in the maxillary arch (in $\mathrm{mm}$ ).

\begin{tabular}{lll}
\hline Operator 1 & Operator 2 & Differential \\
\hline 51.99 & 51.54 & 0.45 \\
53.29 & 53.63 & 0.34 \\
57.92 & 58.54 & 0.62 \\
49.45 & 49.76 & 0.31 \\
57.83 & 57.89 & 0.06 \\
49.55 & 49.60 & 0.05 \\
56.48 & 56.01 & 0.47 \\
54.29 & 54.53 & 0.24 \\
51.66 & 51.66 & 0.00 \\
45.55 & 44.98 & 0.57 \\
& & Mean: 0.01
\end{tabular}

Table 5: Inter-operator error in the mandibular arch (in $\mathrm{mm}$ ).

\begin{tabular}{lll}
\hline Operator 1 & Operator 2 & Differential \\
\hline 54.92 & 54.85 & 0.07 \\
46.61 & 46.84 & 0.23 \\
52.52 & 52.30 & 0.22 \\
44.35 & 43.71 & 0.64 \\
51.40 & 50.91 & 0.49 \\
43.62 & 43.55 & 0.07 \\
48.88 & 48.91 & 0.03 \\
48.45 & 48.07 & 0.38 \\
45.49 & 45.36 & 0.13 \\
40.20 & 40.21 & 0.01 \\
& & Mean: 0.227
\end{tabular}

Using the statistical applications mentioned above, the ICC for the maxillary and mandibular arches are as follows:

\begin{tabular}{lll}
\hline ICC values & Intra-operator & Inter-operator \\
\hline Maxilla & 0.996 & 0.996 \\
Mandible & 0.993 & 0.998 \\
\hline
\end{tabular}

\section{DISCUSSION}

The above results demonstrate the high correlations for both intra-operator and inter-operator dimensions of inter-molar distances. An ICC reading of greater than 0.75 is indicative of good reliability and an ICC of 1 is an identical match in measurements. Studies have also indicated that digital models are a more reliable and clinically acceptable method of assessing inter-molar and inter-canine distances [5, 13-16]. 
In the literature, it is suggested that variations can arise in measurements relating to the operator position and point chosen on the digitized casts. However, as in our study, the variation is often less than that of caliper hand measurements [13].

\section{CONCLUSION}

The above results have shown that three-dimensional scanning techniques are an appropriate method of assessing dental arch morphology than using digital calipers to measure dental arch casts, which provides more extensive measurements. The preliminary results of this study demonstrate that it is viable for future detailed analysis of the dental arch morphology in this sample.

\section{ACKNOWLEDGEMENTS}

We are grateful for the participation of the patients in this study, we would also like to acknowledge the assistance and advice of Dr M Sassani and Dr D Haag. We are also grateful to the University of Otago dental laboratory staff for their patience and assistance with this study.

\section{REFERENCES}

[1] Brook, A.H., O’Donnell, M.B., Hone, A., Hart, E., Hughes, T.E., Smith, R.N. \& Townsend, G.C., General and craniofacial development are complex adaptive processes influenced by diversity. Australian Dental Journal, 59, pp. 13-22, 2014. https://doi.org/10.1111/adj.12158

[2] Wang, J., Sun, K., Shen, Y., Xu, Y., Xie, J., Huang, R., Zhang, Y., Xu, C., Zhang, X., Wang, R. \& Lin, Y., DNA methylation is critical for tooth agenesis: implications for sporadic non-syndromic anodontia and hypodontia. Scientific Reports, 6, 19162, 2016. https://doi.org/10.1038/srep19162.

[3] Al Shahrani, I., Togoo, R.A. \& AlQarni, M.A., A review of hypodontia: Classification, prevalence, etiology, associated anomalies, clinical implications and treatment options. World Journal of Dentistry, 4(2), pp. 117-125, 2013. https://doi.org/10.5005/jp-journals-10015-1216

[4] Thesleff I., Current understanding of the process of tooth formation: transfer from the laboratory to the clinic. Australian Dental Journal, 59, pp. 48-54, 2014. https://doi.org/10.1111/adj.12102

[5] Brook, A.H., Koh, K. \& Toh, V., Influences in a biological complex adaptive system: environmental stress affects dental development in a group of Romano-Britons. International Journal of Design \& Nature and Ecodynamics, 11(1), pp. 33-40, 2016. https://doi.org/10.2495/dne-v11-n1-33-40

[6] Koh, K., Toh, V., Brook O’Donnell, M., Ranjitkar, S. \& Brook, A.H., A complex adaptive system in which environmental stress affects gene expression during development. International Journal of Design \& Nature and Ecodynamics, 11(4) pp. 686-695, 2016. https://doi.org/10.2495/dne-v11-n4-686-695

[7] Lam, F., Yong, R., Ranjitkar, S., Townsend, G.C. \& Brook, A.H., Agents within a developmental complex adaptive system: intrauterine male hormones influence human tooth size and shape. International Journal of Design \& Nature and Ecodynamics, 11(4), pp. 696-702, 2016.

https://doi.org/10.2495/dne-v11-n4-696-702 
[8] Brook A.H., Jernvall J., Smith R.N., Hughes T.E. \&Townsend G.C., The dentition: the outcomes of morphogenesis leading to variations of tooth number, size and shape. Australian Dental Journal, 59(S1), pp. 131-142, 2014. https://doi.org/10.1111/adj.12160

[9] Polder, B.J., Van't Hof, M.A., Van der Linden, F.P. \& Kuijpers-Jagtman A.M., A meta-analysis of the prevalence of dental agenesis of permanent teeth. Community Dentistry and Oral Epidemiology, 32(3), pp. 217-226, 2004. https://doi.org/10.1111/j.1600-0528.2004.00158.x

[10] Brook, A.H., Multilevel complex interactions between genetic, epigenetic and environmental factors in the aetiology of anomalies of dental development. Archives Oral Biology, 54S, pp. S3-S17, 2009. https://doi.org/10.1016/j.archoralbio.2009.09.005

[11] Khalaf K., Miskelly J., Voge E. \& Macfarlane T.V., Prevalence of hypodontia and associated factors: a systematic review and meta-analysis. Journal of Orthodontics, 41(4), pp. 299-316, 2014. https://doi.org/10.1179/1465313314Y.0000000116

[12] Stevens, D., Mir, C., Nebbe, B., Raboud, D., Heo, G. \& Major, P., Validity, reliability, and reproducibility of plaster vs digital study models: Comparison of peer assessment rating and Bolton analysis and their constituent measurements. American Journal Orthodontics and Dentofacial Orthopaedics, 129, pp. 794-803, 2006. https://doi.org/ 10.1016/j.ajodo.2004.08.023

[13] Bell, A., Ayoub, A. \& Siebert, P., Assessment of the accuracy of a three- dimensional imaging system for archiving dental study models. Journal of Orthodontics, 30, pp. 219-223, 2003. https://doi.org/10.1093/ortho/30.3.219

[14] Moreira, D., Gribel, B., Torres, G., Vasconcelos, K., Freitas, D. \& Ambrosano, G., Reliability of measurements on virtual models obtained from scanning of impressions and conventional plaster models. Brazilian Journal Oral Science, 13(4), pp. 297-302, 2014. https://doi.org/10.1590/1677-3225v13n4a11

[15] Harris, E.F. \& Smith, R.N., Accounting for measurement error: a critical but often overlooked process. Archives of Oral Biology, 54(1), pp. 107-117, 2008. https://doi.org/10.1016/j.archoralbio.2008.04.010

[16] Cicchetti, D., Guidelines, criteria, and rules of thumb for evaluating normed and standardized assessment instruments in psychology. Psychological Assessment, 6(4), pp. 284-290, 1994.

https://doi.org/10.1037//1040-3590.6.4.284 